Jpn. J. Med. Sci. Biol., 47, 127 - 139, 1994.

\title{
ELECTRON MICROSCOPIC STUDY OF THE DISTRIBUTION AND THE VERTICAL TRANSMISSION OF RICKETTSIA TSUTSUGAMUSHI IN LEPTOTROMBIDIUM PALLIDUM
}

Hiroshi URAKAMI, Mamoru TAKAHASHI1, Michisato MURATA2 and Akira TAMURA

Niigata College of Pharmacy, 5-13-2 Kamishin'ei-cho, Niigata, Niigata 950-21, 1Kawagoe Agricultural Senior High School, 5-14 Kosenba-cho, Kawagoe, Saitama 350 and 2Jichi Medical School, 3311-1 Minamikawachi-cho, Yakushiji, Kawachi-gun, Tochigi 329-04

(Received May 9, 1994. Accepted July 5, 1994)

SUMMARY: Leptotrombidium pallidum naturally infected with Rickettsia tsutsugamushi was reared and bred in our laboratory for several generations by brother and sister mating. The larvae and adults at the 8 th and 9 th generations were sectioned and observed by electron microscopy for analysis for the distribution of rickettsiae in the mites. The distribution densities of rickettsiae were markedly different among organs in each mite, but rickettsiae were seen in all the organs and tissues. Rickettsiae were distributed in the highest density in the salivary gland of larvae, and in the salivary gland, excretory bladder, epidermal layer, ovary and testis of adult mites. Only a few rickettsiae were recognized in the muscle of both larvae and adults. On the other hand, we found, in the infected family line used, a significant number of mites in which no rickettsiae were found by electron microscopy. The grouping of rickettsia-positive and -negative mites according to the parent family revealed that the efficiency of vertical transmission of rickettsia was different from one parent family to another. Thus, it became clear that a significant number of rickettsia-negative mites are produced in an infected family line.

浦上 弘·多村 憲(新潟薬科大学微生物学教室 新潟市上新栄町5-13-2)

高橋 守(川越農業高等学校＼cjkstart埼玉県川越市小仙波町5-14)

村田道里(自治医科大学実験医学センター 栃木県河内郡薬師寺南河内町3311-1) 


\section{INTRODUCTION}

Some species of trombiculid mites are known to be infected with an endosymbiont, Rickettsia tsutsugamushi, which causes a human febrile disease, scrub typhus or so-called tsutsugamushi disease (10). The ratio of infected mites to uninfected ones varies from one rickettsia-containing species to another, but, in all the cases, the population of mites infected with rickettsia is usually lower than that of uninfected mites. Several investigators succeeded in rearing rickettsiainfected mites in the laboratory and demonstrated transovarial transmission of the rickettsiae from the female parents to the progeny $(2,3)$. In the rickettsiainfected families, most of the progeny contained rickettsiae, but some uninfected progeny were also found $(2,3,5)$. On the other hand, the distribution of rickettsiae in the body of these infected mites has been observed in Leptotrombidium fletcheri by immunofluorescent microscopy (6), and in L. arenicola by electron microscopy (14). The rickettsiae were distributed widely in various organs and tissues of infected mites, most densely in the salivary gland of unfed larvae and the ovary of adult females.

We succeeded also in establishing infected family lines of $L$. pallidum, which is considered as a main vector of $R$. tsutsugamushi in Japan, and maintained them in our laboratory (9). The family lines of $L$. pallidum were distinct from the other species of Leptotrombidium mites in that both male and female progeny were produced at a 1:1 ratio not only in the uninfected but also in the rickettsia-infected family lines $(8,9)$, in contrast to the other species, $L$. fletcheri, $L$. arenicola and $L$. deliense, in which only female progeny were produced in the infected families (4). In the infected family lines of $L$. pallidum, many progeny contained rickettsiae, but some did not (8).

Our previous studies by electron microscopy demonstrated that the rickettsiae in infected female adults of $L$. pallidum were vertically transmitted to the progeny through the eggs, but the rickettsiae in the spermatogonia of infected males were eliminated from the cells during spermatogenesis and were not transmitted to the progeny (12). In the course of these electron microscopic studies, we found that rickettsiae were distributed in different densities in various organs and tissues of mites. In the present study, the distribution densities of rickettsiae in the organs and tissues of the larval and adult $L$. pallidum were analyzed by electron microscopy. Furthermore, we found that rickettsia-positive and -negative mites could be clearly distinguished by electron microscopic observation. Therefore, the production rate of rickettsia-positive progeny in the 
rickettsia-infected family line was also analyzed, and the symbiosis of rickettsiae in the Leptotrombidium was examined.

\section{MATERIALS AND METHODS}

Mites: Four rickettsia-infected family lines of $L$. pallidum were established from engorged larvae which were recovered from wild rodents captured in Saitama Prefecture and were bred by brother and sister mating as mentioned previously $(8,9)$. The symbionts in the four family lines were identified as $R$. tsutsugamushi, Karp type, from the reactivity to strain-specific monoclonal antibodies (9). The mites, unfed larvae and adults, at the 8th and 9th generations in one of the infected family lines were used for the present study.

Electron microscopy: Mites, eggs and spermatophores were prepared for electron microscopy as mentioned previously $(11,12)$. Briefly, samples were fixed doubly with $2 \%$ glutaraldehyde and $1 \%$ osmic acid in $0.1 \mathrm{M}$ cacodylate buffer, $\mathrm{pH}$ 7.4 , stained en bloc with $2 \%$ aqueous uranyl acetate, dehydrated in a graded of ethanol series, and embedded in an Araldite-Epon mixture. Ultrathin sections were mounted on grids coated with formvar and carbon, stained with a lead solution, and observed under a JEOL 100CX electron microscope.

Determination of distribution density of rickettsiae in organs and tissues of mites: The distribution density of rickettsiae in the organs and tissues of each mite was determined by observing two to three sections of different parts of a mite. Electron micrographs of the sections at a magnification of 4,000 times were covered with a transparent section paper ruled into $1.6-\mathrm{cm}^{2}$ squares (corresponds to $10 \mu \mathrm{m}^{2}$ in the electron micrographs), and the size of area of each organ or tissue in $\mu \mathrm{m}^{2}$ was determined by counting the squares of section paper on each organ or tissue. The number of rickettsiae in each organ or tissue was recorded and the distribution density of rickettsiae was expressed as the number of rickettsiae per $100 \mu \mathrm{m}^{2}$.

Discrimination between rickettsia-positive and -negative mites by antirickettsial antibody production in mice: One or two larval mites were homogenized with a mortar and a pestle in SPG (1), and the homogenate was injected intraperitoneally into a ddY mouse. Fourteen days after injection, the spleen and serum were harvested and the homogenates of the spleens were passaged to other mice. This passage was repeated at least twice if anti-rickettsial antibody was not detected in the serum. Antibody was titrated by the indirect immunofluorescence method with Karp strain propagated in L cells as the antigen (9), and the serum showing a titer higher than 1:10 was judged as antibody-positive. 


\section{RESULTS}

\section{Distribution of Rickettsiae in Larval and Adult Mites}

Several organs were identified in larval L. pallidum, such as the salivary gland, tubular gland, midgut, excretory bladder, brain and the rudiment of the reproductive organ, and the tissues such as muscle, nerve, hemolymph and epidermal layers (Fig. 1a). The same organs and tissues were identified in adult $L$. pallidum except the replacement of the rudiments to the mature reproductive organs. Rickettsiae were found in all these organs and tissues. No rickettsiae or rickettsia-like structures were found in the uninfected family lines.

To know the distribution of rickettsiae in each organ and tissue and to compare the distribution profiles of larvae and adults, the rickettsiae per $100 \mu \mathrm{m}^{2}$ in each organ or tissue were counted by the method described in the section of Materials and Methods (Tables I and II). In larvae, rickettsiae were located most densely in the salivary gland or in the tubular gland. In the adults, the rickettsial distribution was seen in high density in the salivary gland, excretory bladder, epidermal layer, ovary and testis. The ovary and testis in adults contained a comparable number of rickettsiae to that in the salivary gland. The midgut, the largest organ in both larvae and adults, and brain contained a moderate number of rickettsiae. In the muscle, only a few rickettsiae were found in many larvae and adults, while some mites had a moderate number of rickettsiae in this tissue.

Comparison of the results in Tables I and $\Pi$ revealed that the density of rickettsiae is higher in the excretory bladder, epidermal layer and reproductive organs of the adult mites than in those of the larval mites. All the organs and tissues in adults are much larger in volume than those in larvae, indicating that the total number of rickettsiae increases during the developmental cycle from larvae to adults. Eggs after oviposition contained 0.78 rickettsiae per $100 \mu \mathrm{m}^{2}$ (average of five eggs), when analyzed by the same method. No rickettsiae were found in spermatophores deposited by infected males $\left(<0.05 / 100 \mu \mathrm{m}^{2}\right)$. This observation on the spermatophores is in agreement with our previous observation that the rickettsiae in males are not transmitted to the spermatophores $(8,9)$.

The distribution pattern of rickettsiae in organs and tissues showed the tendency mentioned above, but the distribution density of rickettsiae in each organ and tissue does differ greatly from one mite to another as Tables I and II show. For example, more than a 100 -fold difference is seen between the salivary gland of No. 5 and that of No. 9 larvae, and between the epidermal layer of No. 1 and 


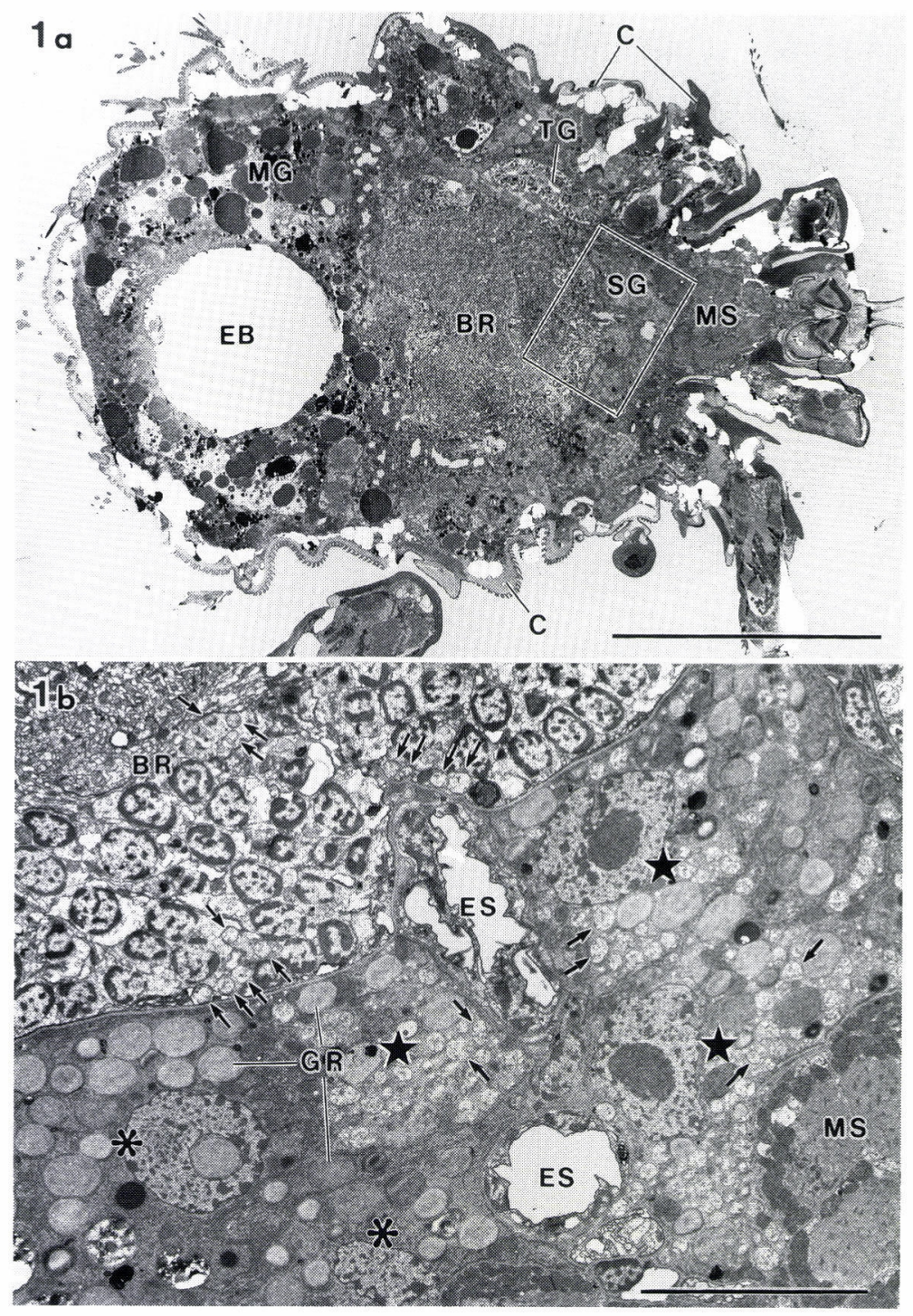

Fig. 1a. A horizontal section of a larva. BR: brain, C: cuticle, EB: cavity of excretory bladder, MG: midgut, MS: muscles, SG: salivary glands, TG: tubular gland. scale $=50 \mu \mathrm{m}$.

Fig. 1b. A higher magnification of a part of Fig. 1a indicated by rectangle. The cells indicated by stars in the salivary gland (SG) contain a number of rickettsiae (arrows), but none in the other cells indicated by asterisks. All the rickettsiae in the brain (BR) are indicated by arrows. ES: esophagus, GR: salivary granules, MS: muscles. scale $=10 \mu \mathrm{m}$. 


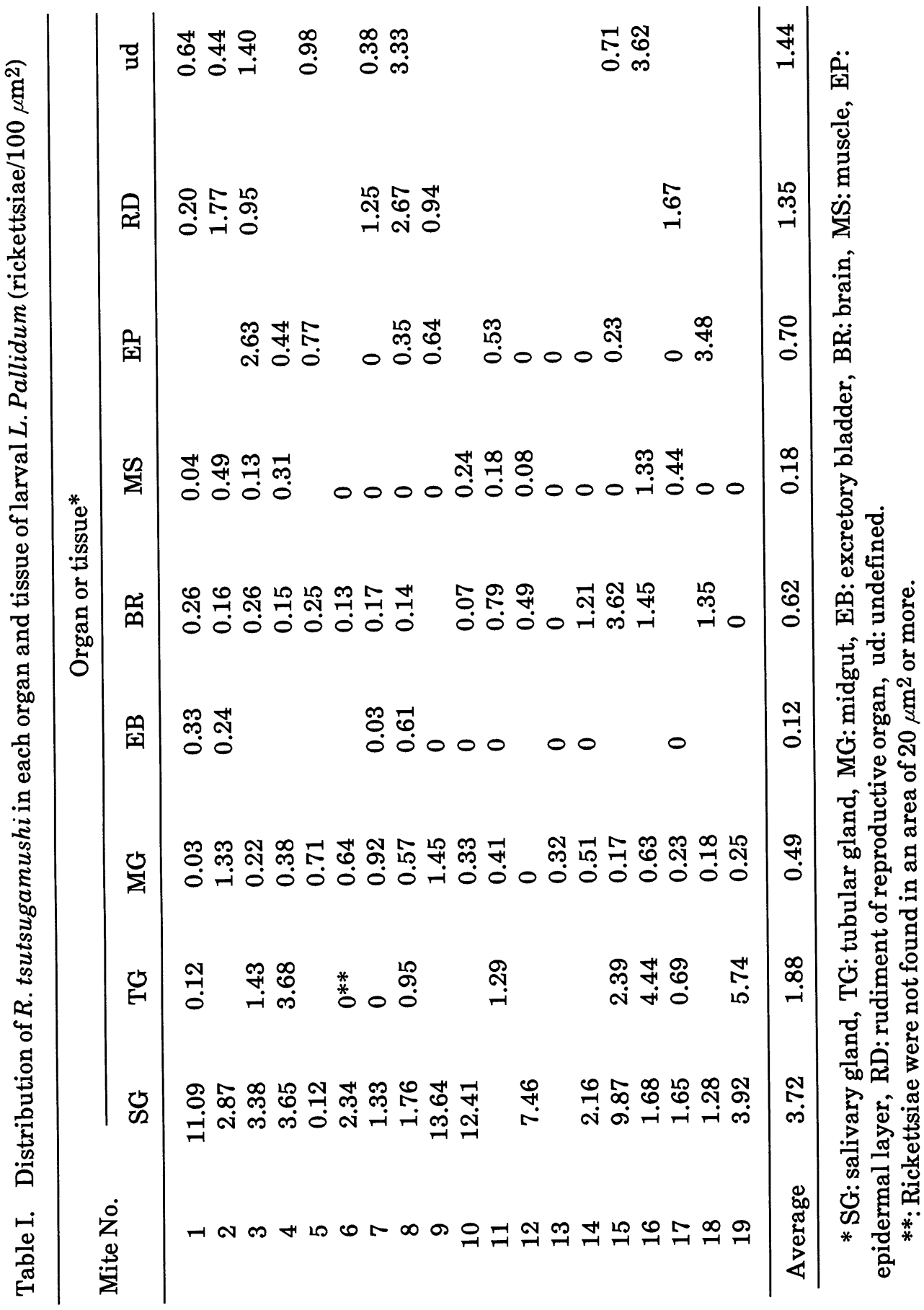




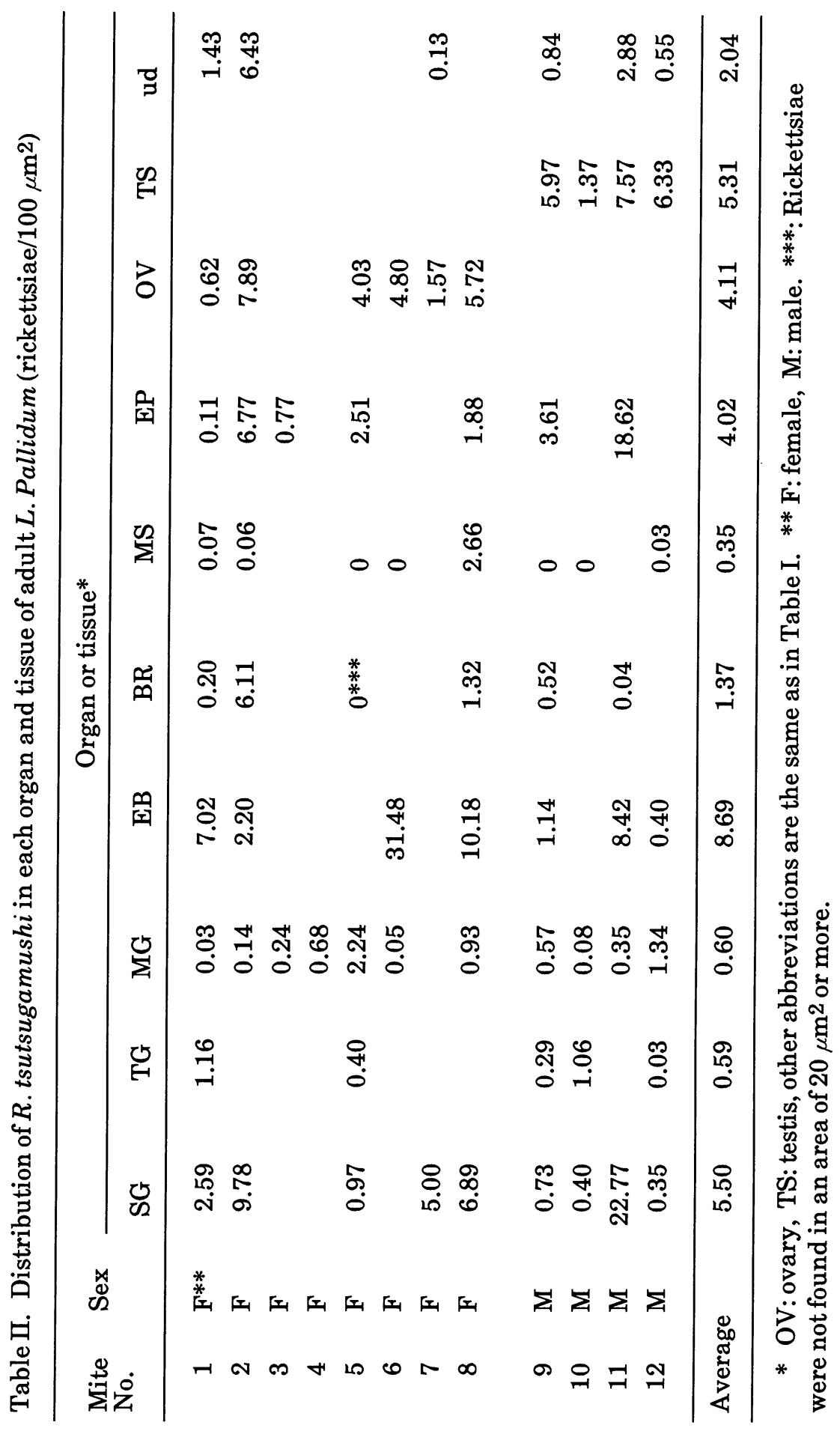



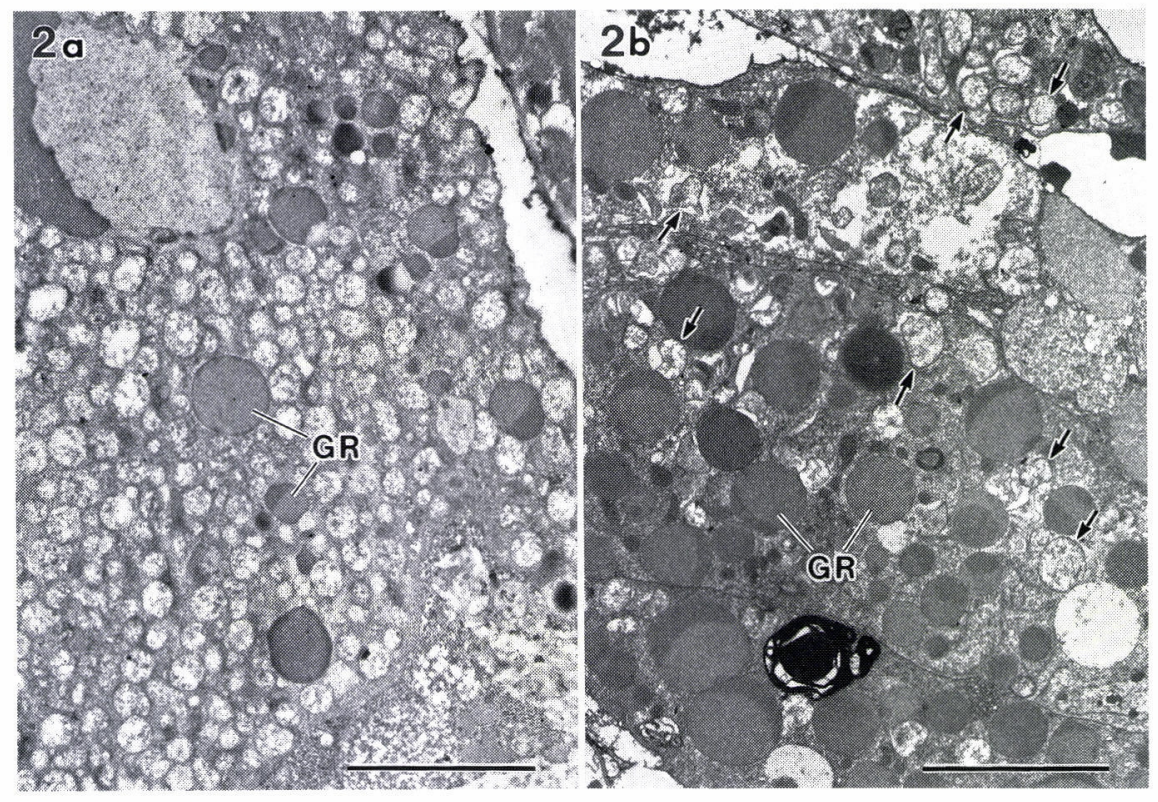

Fig. 2a and b. Salivary glands of adults Nos. 11 and 12 in Table II, respectively. Cytoplasm is filled with rickettsiae (arrows) in Fig. 2a, but rickettsiae are sparsely seen in Fig. 2b. GR: salivary granules. scale $=5 \mu \mathrm{m}$.

that of No. 11 adults. Figure 2 shows the distribution of rickettsiae in salivary glands of adults Nos. 11 and 12 in Table $\Pi$. The densities of the microorganisms are clearly different between these two profiles. The adult mites which have high values in the salivary glands, show relatively high densities also in the other organs (Nos. 2, 8 and 11 in Table II), suggesting that the total numbers of rickettsiae in the whole body are greatly different from one mite to another.

Because the observations were performed on thin sections of the samples, it is unclear whether rickettsiae are distributed evenly in the whole organ. At the cellular level, unequal distribution is clearly seen in Fig. 1b. In the salivary gland zone of this figure, many rickettsiae are seen in the cytoplasm of the cells indicated with stars, but none in the cells indicated with asterisks. Similarly, some cells in the brain zone of the same figure contain several rickettsiae but the others do not. 


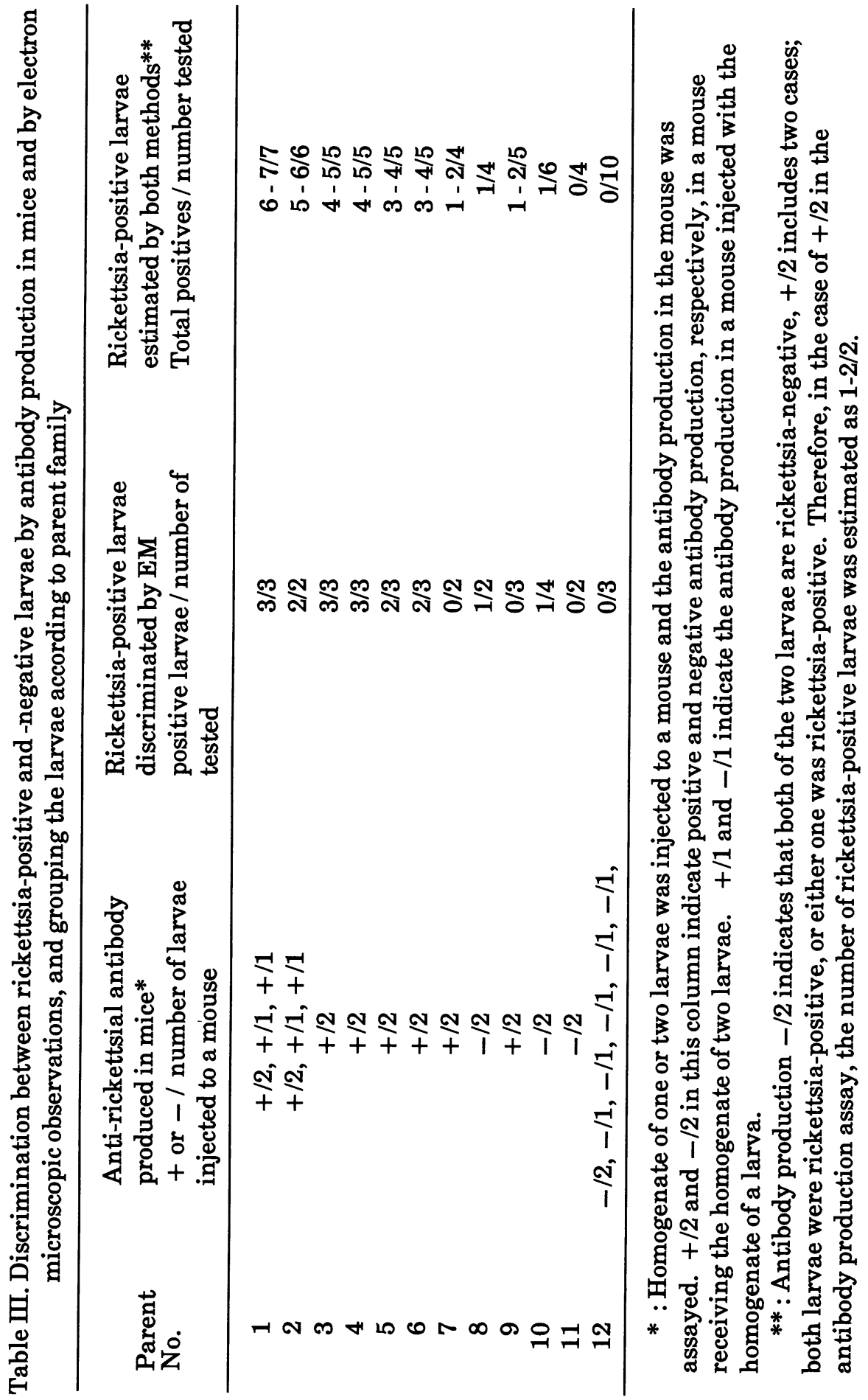




\section{Ratio of Rickettsia-positive and-negative Mites at the 8th or 9th Generation of an Infected Family Line}

In the present study, we used the mites at the 8th or 9th generation of the established family line. This family line originated from a pair of an infected female and an uninfected male, and was maintained by brother and sister mating. The rate of infected progeny at the first three generations was calculated at $97 \%$ (8). However, in the course of this study, we found some mites containing no rickettsiae by electron microscopy. Therefore, the rate of infected progeny at the $8 \mathrm{th}$ and 9 th generations was checked again by detecting the anti-rickettsial antibody produced in mice after the injection of a homogenate of one or two larvae. On the other hand, because the rickettsiae are distributed widely in mites as mentioned above, the rickettsia-positive and -negative mites are easily discriminated by the electron microscopic observation of thin-sectioned specimens of mites. Electron microscopic examinations for discrimination between rickettsia-positive and -negative larvae were performed independently from the assay for the antibody producted. Table III, in which the larvae tested are listed according to the parent family having produced the larvae, shows the results obtained by both methods. The families were classified into three groups, rickettsia-positive progeny were dominantly produced (parent Nos. 1-4), both rickettsia-positive and -negative progeny were produced in variable ratios (parent Nos. 5-10), and almost all progeny were rickettsia-negative (parent Nos. 10-11). This indicates that the uninfected progeny were produced at a significant rate in the infected family line during maintenance by brother and sister mating, and that the efficiencies of production of rickettsia-positive progeny were different from one parent to another.

\section{DISCUSSION}

The distribution of $R$. tsutsugamushi in various organs and tissues of $L$. fletcheri was first observed by immunofluorescent microscopy (6). Wright et al. (4) also observed $L$. arenicola naturally infected with the rickettsia by electron microscopy, and reported wide distribution of rickettsiae in almost all organs and tissues except the muscles. They also reported that rickettsiae were distributed most densely in the salivary glands of larvae and in the ovary of adult female mites. In our electron microscopic observation of infected L. pallidum reared in 
our laboratory, rickettsiae were distributed in all organs and tissues of both larvae and adults including the muscles, although there were only a few rickettsiae in the muscle. In the larvae, the salivary gland contained rickettsiae most densely among the organs as in L. arenicola. However, in adults of L. pallidum, a significant number of rickettsiae were distributed in the reproductive organs but, in many cases, a higher concentration of rickettsiae was seen in the salivary glands, excretory bladders or epidermal layers.

In the results shown in Tables I and II, extremely high concentrations of rickettsiae were observed in the organs of some mites (see also Fig. 2a), which may have occurred by chance on thin sections, but the mites which had many rickettsiae in some organs showed relatively high concentrations of rickettiae in the other organs and tissues. This indicates that these mites may have had a larger number of rickettsiae in their whole body than the other mites. In other words, it may be true that the number of rickettsiae may differ markedly from one infected mite to another.

Figure $1 \mathrm{~b}$ shows that some cells in the salivary gland had many rickettsiae but the neighboring cells had none. This suggests infrequent migration of rickettsiae from cells to the neighbors in the mites. Budding of rickettsiae, a release form of rickettsia from the host cell surface, was rarely seen in the mites $(11,12)$, also suggesting the infrequent migration of rickettsiae from cells to others.

In our infected family line of mites, a significant number of rickettsianegative mites were detected. The families were classified into three groups according to parent-children correlation. As mentioned above, the number of rickettsiae in mites may be different among individual mites. Therefore, the different concentrations of rickettsiae in the parents may be related to the different efficiency of production of rickettsia-positive and -negative progeny: the parents which have a large number of rickettsiae in their body produce rickettsia-positive progeny at a high probability and those which have fewer rickettsiae in the body produce a significant number of rickettsia-negative progeny. Figure $1 \mathrm{~b}$ shows that the distribution and contents of rickettsiae in individual cells vary even among the cells of the same organ. Therefore, the distribution of rickettsiae in oocysts may be uneven and, if a female parent contains only a small number of rickettsiae in the ovary, the female will produce rickettsia-negative eggs in a high efficiency and it will result in the production of many rickettsia-negative progeny.

Rapmund et al. (3) reported that the rate of rickettsia-positive mites in an infected family of $L$. fletcheri was $98 \%$. In L. arenicola, Roberts et al. (6) observed that the rate varied from 20 to $100 \%$, depending on the female parents. In our in- 
fected family line of $L$. pallidum, the infection rate was about $97 \%$ in the first three generations (8). After that, the rate was not assayed for several generations, and in the present study, decrease in the rate to about $50 \%$ was found at the 8th and 9 th generations. For maintenance of the infected family lines, it is necessary to analyze the infection rate periodically and to select such families as producing rickettsia-positive progeny at a high efficiency.

Roberts et al. (7) reported that uninfected females produced more eggs than the infected ones in L. arenicola, but fewer in L. fletcheri. In L. pallidum, we found no clear difference in the life span, egg production, or hatchability rate between the infected and uninfected family lines. It is still unknown at present, however, whether an extremely high concentration of rickettsiae in organs has any effect on the mite's life cycle.

\section{REFERENCES}

1. Bovarnick, M. R., Miller, J. C. and Snyder, J. C. (1950): The influence of certain salts, amino acids, sugars and proteins on the stability of rickettsiae. J. Bacteriol., 59, 509-522.

2. Rai, J. and Bandopadhyay, D. (1978): Vertical transmission in chiggerborne rickettsiosis. Indian J. Med. Res., 68, 31-38.

3. Rapmund, G., Upham, R. W., Jr., Kundin,-W. D., Manikumaran, C. and Chan, T. C. (1969): Transovarian development of scrub typhus rickettsiae in a colony of vector mites. Trans. Roy. Soc. Trop. Med. Hyg., 63, 251-258.

4. Rapmund, G. (1984): Rickettsial disease of the far east: new perspectives. J. Infect. Dis., 149, 330-338.

5. Roberts, L. W. and Robinson, D. M. (1977): Efficiency of transovarial transmission of Rickettsia tsutsugamushi in Leptotrombidium arenicola (Acari: Trombiculidae). J. Med. Entomol., 13, 493-496.

6. Roberts, L. W., Robinson, D. M., Rapmund, G., Walker, J. S., Gan, E. and Ram, S. (1975): Distribution of Rickettsia tsutsugamushi in Leptotrombidium (Leptotrombidium) fletcheri (Prostigmata: Trombiculidae). J. Med. Entomol., 12, 345-348.

7. Roberts, L. W., Rapmund, G. and Cadigan, F. C., Jr. (1977): Sex ratios in Rickettsia tsutsugamushi-infected and noninfected colonies of Leptotrombidium (Acari: Trombicuidae). J. Med. Entomol., 14, 89-92.

8. Takahashi, M. (1990): Vertical transmission of Rickettsia tsutsugamushi in Leptotrombidium (Leptotrombidium) pallidum. Jpn. J. Sanit. Zool., 41, 389403 (text in Japanese). 
9. Takahashi, M., Murata, M., Nogami, S., Hori, E., Kawamura, A., Jr. and Tanaka, H. (1988): Transovarial transmission of Rickettsia tsutsugamushi in Leptotrombidium pallidum successively reared in the laboratory. Jpn. J. Exp. Med., 58, 213-218.

10. Tamiya, T. (1962): Recent advances in studies of tsutsugamushi disease in Japan. Medical Culture Inc., Tokyo. 309 p.

11. Urakami, H., Takahashi, M., Tamura, A. and Hori, E. (1988): Electron microscopic observations of the embryo Leptotrombidium (Leptotrombidium) pallidum naturally infected with Rickettsia tsutsugamushi. Microbiol. Immunol., 32, 967-972.

12. Urakami, H., Takahashi, M., Hori, E. and Tamura, A. (1994): An electron microscopic study of vertical transmission of Rickettsia tsutsugamushi in the oogenesis and athe spermatogenesis in Leptotrombodium pallidum. Am. J. Trop. Med. Hyg., 50, 219-228.

13. Urakami, H., Tsuruhara, T. and Tamura, A. (1984): Electron microscopic studies on intracellular multiplication of Rickettsia tsutsugamushi in L cells. Microbiol. Immunol., 28, 1191-1201.

14. Wright, J. D., Hastriter, M. W. and Robinson, D. M. (1984): Observations on the ultrastructure and distribution of Rickettsia tsutsugamushi in naturally infected Leptotrombidium (Leptotrombidium) arenicola (Acari: Trombiculidae). J. Med. Entomol., 21, 17-27. 